\title{
Safety Aspects of Vaccinating Premature Infants Against Pertussis and Pneumococci \\ - a Literature Review with Focus on Risk for Apnea and Death
}

C Chrapkowska (1,2), B Aronsson (2), A Lindstrand (2), A-L Johansson (2), S Viksmoen Watle (3), J Storsaeter (3)

(1) Karolinska Institutet, Department of Women's and Children's Health, (2) Swedish Institute of Public Health (3.) Norwegian Institute of Public Health

\section{Learning points}

- $\quad$ Post-vaccination Apnea/ABD events were described in $0-23 \%$ of children born prematurely.

- There was a tendency of higher incidence of apnea/ABD events with lower gestational age at birth (Figure 1).

- No correlation was found between the chronologic age of infants at first vaccination and the occurrence of apnea/ABD events.

- Apnea occurred from 3-66 hours postvaccination.

- $\quad$ Eight fatal cases were reported. No causal relationship between vaccination and death was found.

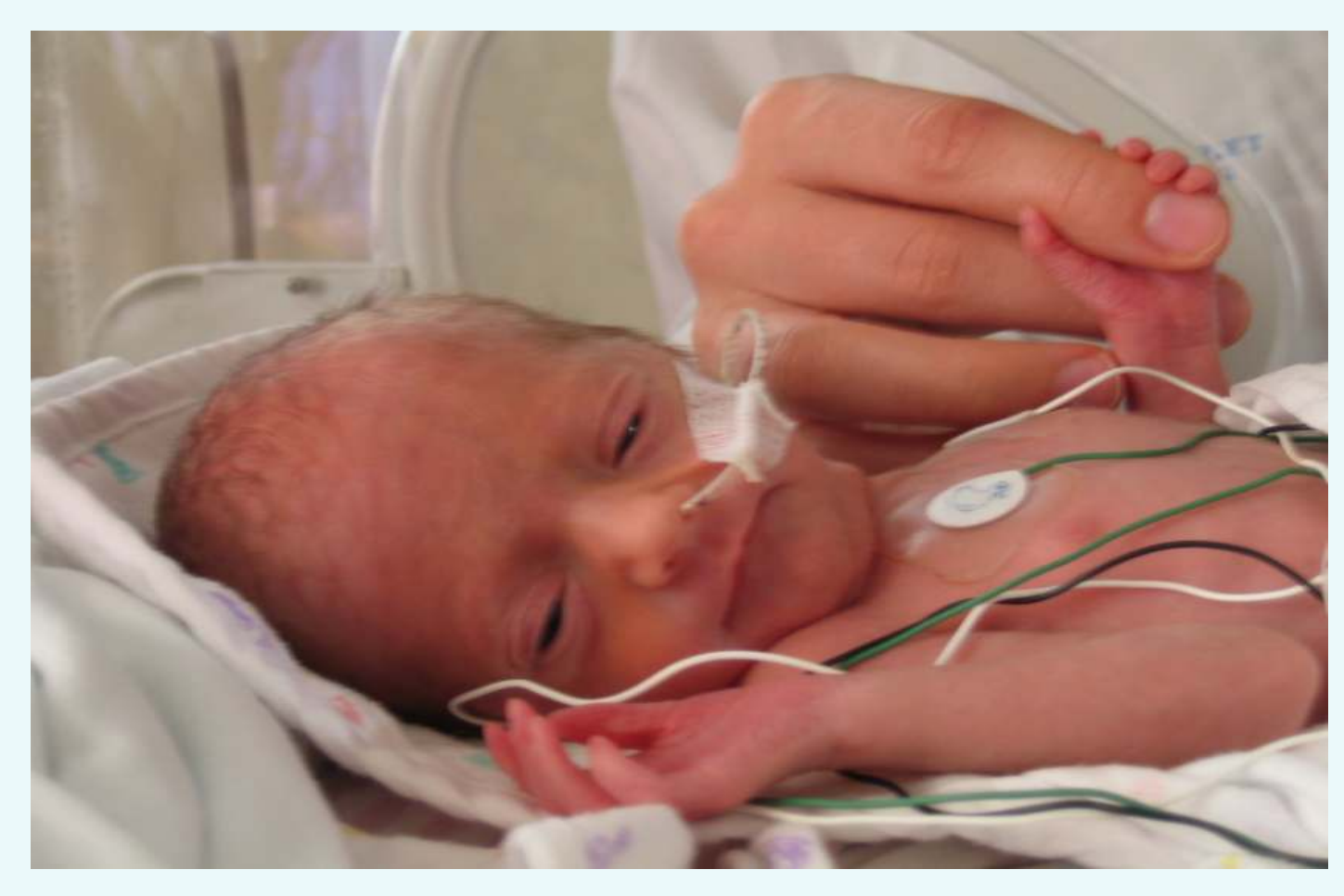

\section{Background}

Premature infants are more vulnerable to vaccinepreventable infections with pertussis and pneumococci. Cardiorespiratory instability is a feared adverse event after immunization of these infants and often leads to deferred vaccination.

We aimed to review the risk of apnea and death following the first vaccination in premature infants. Most studies did not report apnea alone, but in combination with bradycardia and/or desaturation (ABD events).

\section{Methods}

We conducted a literature review of studies published 1986 - 2017. Articles were retrieved from PubMed, Cochrane Library and Scopus. Studies where the outcomes apnea or death were assessed after vaccination with acellular pertussis and/or pneumococcal conjugate vaccines were included.

Of 3679 articles retrieved, 26 were included after reviewing full text, and 8 were included after evaluation with GRADE methodology.

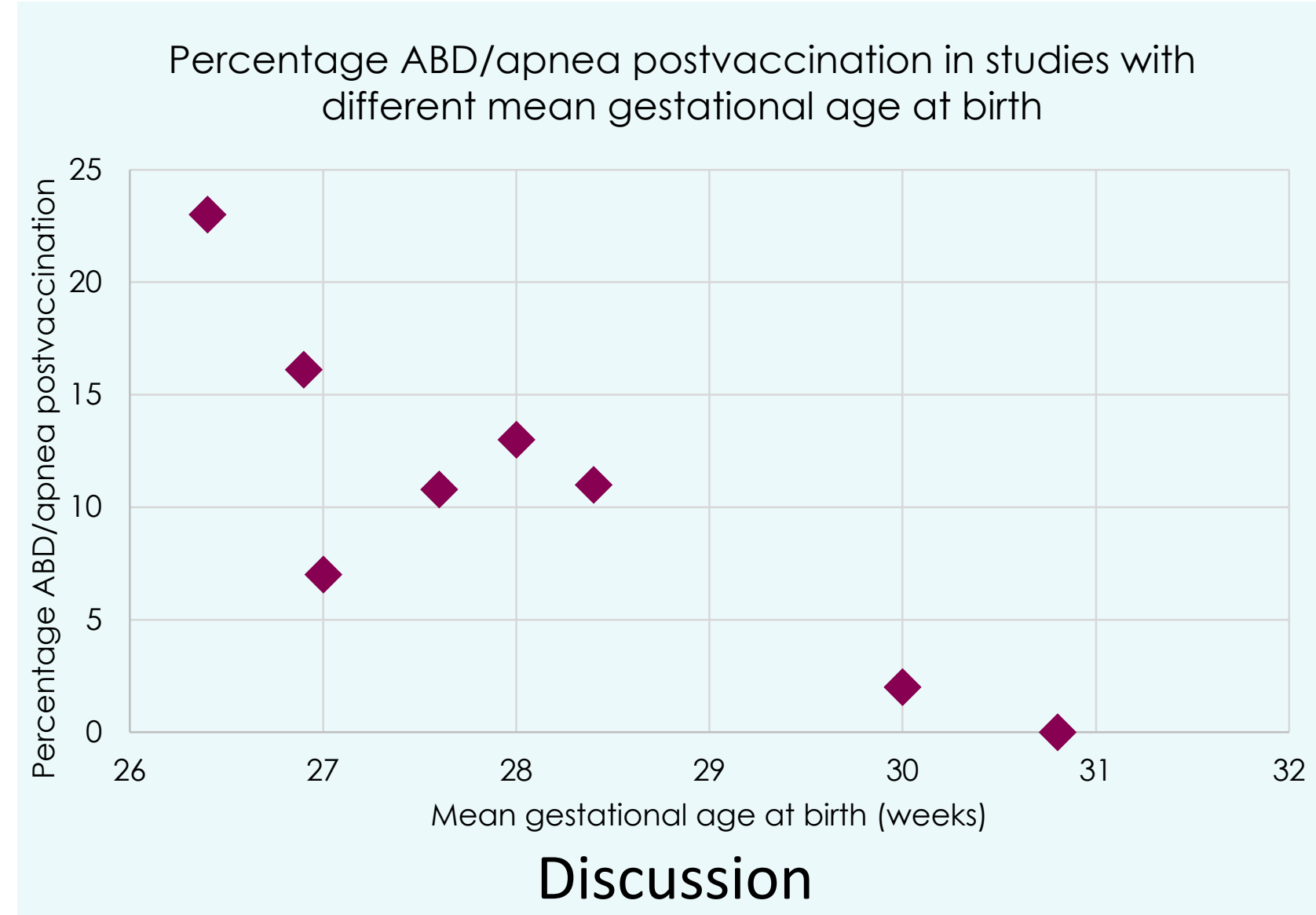

The infants' cardiorespiratory stability and overall clinical condition at the time of vaccination seemed to be of importance for the risk of postvaccination apnea/ABD events.

Percentage of children with increased events varied between studies, largely due to differences in methodology and definitions of outcomes. In the only randomised controlled study in this review, no difference was seen between the vaccinated group and the controls.

One small study showed that pneumococci and hexavalent vaccines given concomitantely gave significantly higher incidence of apnea and bradycardia. There is a need for prospective studies on concomitant vaccination.
Cecilia Chrapkowska, MD, Paediatrician, PhD Student PhD Student • Dept of Women's and Children's Health, Karolinska Institutet

Paediatrician $\bullet$ Unit for Vaccination Programmes Swedish Agency for Public Health Paediatrician, • Alva Paediatric Clinic, Stockholm post: cecilia.chrapkowska@.ki.se Phone: +46-733346637

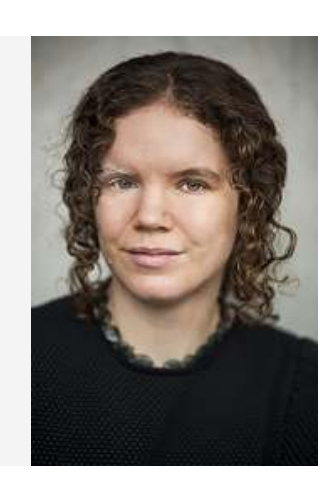

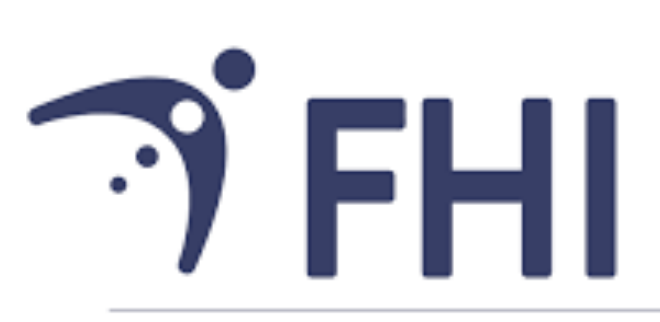

Folkehelseinstituttet

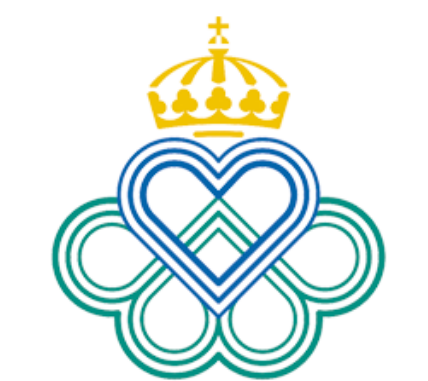

Folkhälsomyndigheten

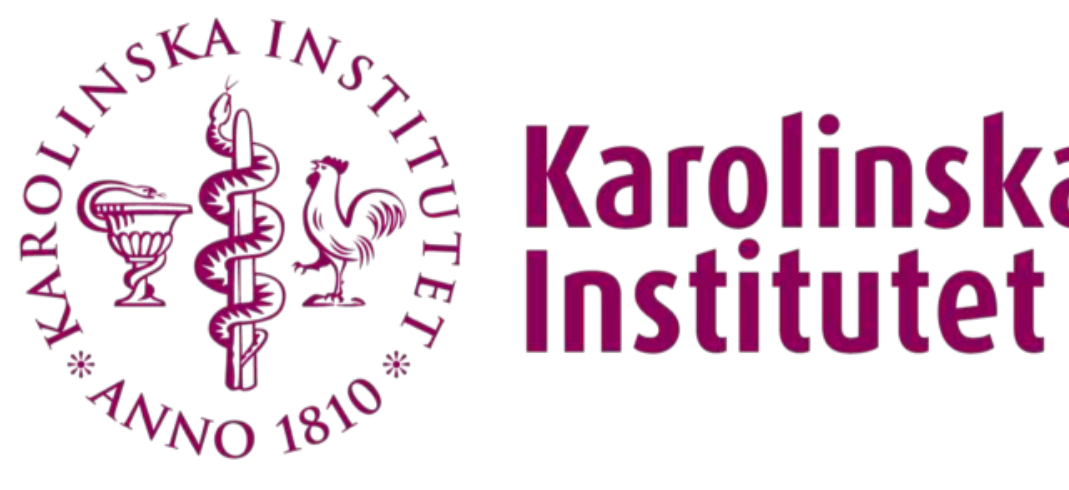

